\title{
Die Colonia Aelia Capitolina: Überlegungen zur ANFANGSPHASE DER ZWEITEN RÖMISCHEN KOLONIE IN DER Provinz Iudaea-Syria Palaestina
}

\author{
Werner Eck \\ Universität zu Köln \\ In memoriam Johann Maier \\ Gründer des Martin-Buber-Instituts \\ für Judaistik der Universität zu Köln \\ 17. Mai 1933-16. März 2019
}

\begin{abstract}
The colonia Aelia Capitolina, founded by Hadrian instead of Jerusalem, was certainly established only after summer 130, as a letter of the emperor to Hierapolis shows, which was written at this time in Jerusalem. One can conjecture who was settled in the colonia at that time, but concrete evidence is hardly to be found in the epigraphic tradition. The territory of the colonia might have been quite large, but cannot be determined in detail. The Roman character of the new city is most evident in the inscriptions, the vast majority of which are written in Latin. The name Aelia is still used for the city well into the Christian era.
\end{abstract}

Keywords: Jerusalem, colonia Aelia Capitolina, Hadrian, veterans, Latin inscriptions.

Das jüdische Jerusalem, wie es sich nach dem Bauboom der herodianischen Zeit dem Besucher präsentierte, muss bis zum Zeitpunkt der Zerstörung durch die römischen Truppen im Jahr 70 n.Chr. ein großes, die Mitwelt beeindruckendes urbanes Zentrum gewesen sein. Dieses Urteil findet sich nicht nur bei Iosephus, der seine Stadt als

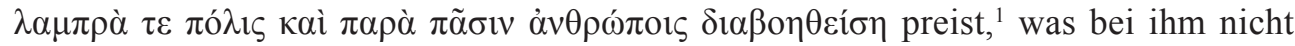
weiter verwunderlich ist. Doch auch römische Autoren lassen den tiefen Eindruck erkennen, den die Stadt auf auswärtige Betrachter gemacht und ihre Berichte entsprechend beeinflusst hat. Plinius der Ältere nennt sie im Kontext seiner Beschreibung der Toparchien in Palaestina longe clarissima urbium Orientis, non Iudaeae modo ${ }^{2}$ und selbst Tacitus spricht in seinem Judenexkurs am Anfang von Buch 5 der Historien von

1 Iosephus, bell. Iud. 7, 4.

2 Plinius, nat. hist. 5, 70. 
einer famosa urbs. ${ }^{3}$ Und noch weit später charakterisiert Eutrop das Jerusalem, das unter Vespasian zusammen mit Iudaea Teil des Reiches geworden war, als urbs nobilissima Palaestinae. ${ }^{4}$

All dies fand mit der Eroberung und Zerstörung der Stadt ein Ende. Die römischen Truppen scheinen sehr gründliche Arbeit geleistet zu haben, auch wenn die neutestamentlichen Aussagen, es würde kein Stein auf dem anderen bleiben, nicht völlig wörtlich genommen werden müssen. ${ }^{5}$ Doch letztlich unterscheiden sich die Aussagen bei Matthäus und Lucas nicht von dem, was Iosephus über die systematische Zerstörung der Stadt durch Titus' Truppen berichtet. ${ }^{6}$ Die Stadt existierte nicht mehr, weder als Siedlung, noch weniger als Gemeinde. Wieweit noch Menschen in den Ruinen lebten, lässt sich nicht sagen. Doch sollten sich die canabae der Legion, die sich notwendigerweise neben dem Lager entwickelten, wenn nicht von Anfang an, so doch zumindest mit der Zeit auch auf das Gebiet des ehemaligen städtischen Raumes ausgedehnt haben. Wie anderswo auch unterstanden sie aber der Befehlsgewalt der Legion und ihres Kommandeurs, der gleichzeitig Statthalter der Provinz war. Ob sich, wie an anderen Legionsstandorten auch, eine Quasiadministration der canabae für die täglichen lokalen Regelungsnotwendigkeiten entwickelte, ${ }^{7}$ lässt sich nicht erkennen. Denn nicht einmal die Existenz der canabae ist dokumentiert, obwohl sie bestanden haben müssen, da keine Legion ohne eine solche Siedlung außerhalb des Lagers längere Zeit überleben konnte. Die jüdische Siedlung, die vor einigen Jahren beim Bau der Straßenbahn in Shu'afat im Norden Jerusalems entdeckt wurde, ${ }^{8}$ hat diese Funktion sicher nicht erfüllen können, da sie vom Lager der legio X Fretensis zu weit entfernt war. Dass sich in den canabae auch Soldaten der Legion nach ihrem Ausscheiden aus dem militärischen Dienst niedergelassen haben, ist mehr als wahrscheinlich. Allerdings lässt sich von den wenigen Soldaten, die durch Inschriften aus Jerusalem bekannt sind, ${ }^{9}$ keiner mit Sicherheit in die Periode zwischen der ersten Stationierung der X Fretensis und der Gründung der colonia Aelia Capitolina durch Hadrian datieren. Mit einiger Wahrscheinlichkeit gehört allein die Grabinschrift des Ti. Claudius Fatalis, eines centurio legionis X Fretensis, in diese Zeit; er starb freilich schon mit 42 Jahren nach nur 23 Jahren Dienst im Heer. Wenn die Datierung zutrifft, dann bezeugt aber gerade sein titulus funebris die Existenz der canabae; denn er wurde von einer Cl(audia) Ionice, die seine lib(erta) et heres war, bestattet. ${ }^{10}$ Diese Freigelassene, die ihn vielleicht schon über mehrere Stationen seiner abwechslungsreichen Laufbahn begleitet hatte, musste irgendwo außerhalb des Lagers leben, wo der centurio sie treffen konnte. Dieses „Paar“ setzt somit zwingend eine Siedlung neben dem Lager voraus. Freilich könnte das Zeugnis auch in die Zeit nach der Gründung der colonia gehören.

3 Tacitus, hist. 5, 2, 1 .

4 Eutropius 7. 19.

5 Siehe Matth. 24, 2; Luc. 19, 44.

6 Iosephus, bell. Iud. 6, 417 ff.; 7, 1 ff.

7 Siehe z.B. CIL III 1008 = ILS 2476 (Apulum): magistra(n)s primus in can(abis); AE 1944, $93=A E$

1955, 9 (Aquincum): dec(urioni) canab [arum].

8 Siehe zu dieser Siedlung Cotton 2007, 12* ff.

9 Siehe CIIP I 2, 721, 732-736.

$10 A E$ 1939, $157=$ CIIP I 2, 734. Siehe auch Dąbrowa 1993, 84, Nr. 7. 
Rund 60 Jahre nach der Zerstörung der Stadt lebte der Name Jerusalem zwar weiter, aber eben nur noch als Bezeichnung für einen Ort, an dem eine römische Legion stationiert war, wie etwa in einer kaiserlichen Konstitution aus dem Jahr 94, wo von Veteranen der legio X Fretensis gesagt wird: qui militaverunt Hierosolym $\{n\}$ is in leg(ione) X Fretense. ${ }^{11}$ Ebenso wird in einem Text, der in den Höhlen von Murabba'at

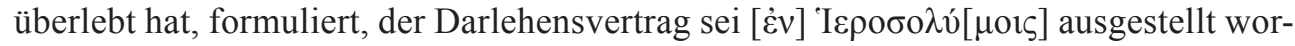
den. Nach aller Wahrscheinlichkeit muss auch dieses Zeugnis vor der Gründung der hadrianischen Kolonie geschrieben worden sein. ${ }^{12}$ Zwischen dem Lager der Legion und Jerusalem musste nicht näher unterschieden werden, da Jerusalem keine eigenständige rechtliche Einheit mehr war, sondern nur noch eine Ortsbezeichnung. Die Legion hatte an diesem Ort ihr Lager. Das zeigt sich e contrario mit aller Klarheit in einem weiteren Darlehensvertrag, der zwischen zwei Soldaten der legio X Fretensis abgeschlossen wurde, nach der Gründung der colonia, vielleicht bald danach. Darin wird klar zwischen dem Lager einerseits und der Kolonie andererseits unterschieden: aput colon[i]am Aeliam Capitolinam ịn hibernis leg(ionis) $\bar{X}$ Frẹt(ensis). ${ }^{13}$ Neben dem Lager existiert wieder eine Stadt, eine sich selbst verwaltende Gemeinde, die den Namen colonia Aelia Capitolina trägt.

Dass Hadrian diese römische Stadt gegründet hat, kann nicht bezweifelt werden; ${ }^{14}$ wohl aber ist der Zeitpunkt und in gewisser Hinsicht auch der Grund dafür umstritten. Man hatte schon stets und nicht ohne Grund die Schaffung der Kolonie mit der Anwesenheit Hadrians in der Provinz im Jahr 130 in Verbindung gebracht, nicht zuletzt deswegen, weil Cassius Dio von der Entscheidung Hadrians für Aelia Capitolina spricht und unmittelbar darauf den Aufstand unter Bar Kochba folgen lässt. ${ }^{15}$ Man darf dies sicher so verstehen, dass für den Historiker der Krieg die Folge dieser Koloniegründung war. Grundsätzlich macht das Sinn, ist jedenfalls weit plausibler als die Behauptung der Historia Augusta, Hadrian habe den Juden die Beschneidung untersagt, was die Erhebung

$11 \quad$ CIL XVI App. $12=$ ILS 9059.

12 P. Mur. 114: Benoit 1957, 260 ff.; dazu Cotton - Eck 2002, 173-183.

13 Bernini 2018, 183-193; cf. Bernini 2019, 557-562. Die genaue Datierung des Papyrus muss offen bleiben; am Anfang des Dokuments steht zwar ein genaues Datum: P[r]isciano et Severo co(n)s(ulibus) $\bar{V}$ Idus Maịas. Doch ein Konsulnpaar mit diesen Cognomina ist bisher nicht bekannt. Zwar erwartet man in einem solchen Dokument in einer Provinz für die Datierung die ordentlichen Konsuln, die generell bekannt sind. Doch in den bekannten Namen der jeweils gemeinsam amtierenden consules ordinarii finden sich diese Cognomina nicht. Möglich wäre, dass einer der Konsuln mehrere Cognomina trug und dass der Schreiber des Dokuments nicht das Hauptcognomen eines Konsuls gewählt hat, wie es manchmal geschieht. Gelegentlich sind auch nicht alle einzelnen Namenselemente eines Senators bekannt.

${ }_{14}$ Aus den neueren Ausgrabungen in der Altstadt von Jerusalem wurden weitgehende Folgerungen für den Zeitpunkt der Gründung der Kolonie gezogen (siehe vor allem Weksler-Bdolah 2014, 38-62). Doch ist nicht zu erkennen, wieso aus Baumaßnahmen, die nach den archäologischen Befunden, allerdings ohne zwingende Faktoren, lediglich in die Zeit vor 130 datiert werden, auf eine Koloniegründung geschlossen werden kann. Daraus ist nicht einmal auf die Absicht einer Koloniegründung zu schließen. Zudem zeigt das im Folgenden angeführte Schreiben Hadrians klar, dass die Kolonie Mitte des Jahres 130 noch nicht bestanden haben kann. Für eine solche Diskrepanz zwischen der angeblichen Planung und baulichen Errichtung einer Kolonie und der weit späteren rechtlichen Gründung müsste man konkrete Belege vorlegen. Bei vielen anderen Kolonien kann man zeigen, dass sie zwar rechtlich gegründet wurden, aber die konkrete bauliche Entwicklung sich danach über einen längeren Zeitraum erstreckte.

15 Cassius Dio 69, 12, $1 \mathrm{f}$. 
provoziert habe. ${ }^{16}$ Dies ist als Strafmaßnahme am Ende des Aufstandes verständlich, nicht aber als einzelnes Verbot ohne einen massiven Anlass.

Seit einigen Jahren kennen wir ein Schreiben Hadrians an die Stadt Hierapolis in Phrygien, das, wie es am Ende des Briefes heißt, $\dot{\varepsilon} v$ 'I $\varepsilon \rho \circ \sigma o \lambda v ́ \mu[o 1 \varsigma]$ abgefasst und von dort abgesandt worden war. ${ }^{17}$ Nun könnte man sagen, Hadrian habe zum Zeitpunkt der Abfassung des Briefes noch nicht die Absicht gehabt, in Jerusalem eine neue colonia zu errichten. Doch dass Hadrian sich spontan von einem Tag zum andern dazu entschlossen habe, ist mehr als unwahrscheinlich. Zudem hielt er sich nicht allzu lange in der Provinz Iudaea auf. Denn im Frühjahr 130 hat er Palmyra besucht, anschließend reiste er durch Arabia und von dort nach Iudaea. Doch bereits im Juli/August ist er in Ägypten angekommen, kann also nicht lange in Iudaea gewesen sein. ${ }^{18}$ Wenn man allerdings die Gründung der Kolonie mit dem Aufenthalt Hadrians in Iudaea verbindet, dann kann zumindest der Gründungsakt, das zeremonielle Umpflügen der colonia, der auch auf späteren Münzen der Stadt mit dem Porträt Hadrians erscheint, ${ }^{19}$ zum Zeitpunkt der Abfassung des Schreibens an die Stadt Hierapolis noch nicht stattgefunden haben. Denn dann wäre auch der neue Name: Aelia Capitolina schon festgelegt gewesen. Somit bleibt offen, ob die Gründung in der kurzen Zeit des Besuchs des Kaisers in der Provinz stattgefunden hat. Dass jedoch Aelia Capitolina der entscheidende Grund für den Aufstand war, bleibt in jedem Fall die wahrscheinlichste Hypothese. Dabei sollte nicht übersehen werden, dass gerade der neue Name der Stadt für Juden besonders provokativ wirken musste. Seit dem Jahr 70 n.Chr. zahlten alle Juden die Steuer, die früher an den Tempel in Jerusalem überwiesen wurde, an den Tempel des Iupiter Capitolinus. Jetzt nahm dieser Iupiter, dem sie weiterhin diese Steuer zu entrichten hatten, auch noch seinen Sitz in ihrem religiösen Zentrum. ${ }^{20}$ Sich gegen diese Demütigung zu wehren, musste vielen Juden fast als religiöse Pflicht erscheinen.

Bisher hatte man weithin den Beginn der Rebellion in den Spätsommer des Jahres 132 datiert. Dabei wurde aber übersehen, dass ein Papyrus, der zu den Bar KochbaDokumenten gehört, eben nach der Ära der Rebellion datiert ist: „,first day of Iyyar, in the first year of the redemption of Israel [by Simeo]n bar Kosiba, Prince of Israel ${ }^{\circ,}{ }^{21}$ was

16 HA v. Hadriani 14, 1: Moverunt ea tempestate et Iudaei bellum, quod vetabantur mutilare genitalia. Dass der Aufstand ausgebrochen sei, weil Hadrian angeblich versprochen habe, den jüdischen Tempel wieder aufzubauen, wie es in spätantiken jüdischen Quellen behauptet wird, muss man als reine Erfindung nicht weiter berücksichtigen. Denn Hadrian hatte selbst während des Diasporaaufstandes erlebt, welche Wucht die jüdische Religion auch im Verhältnis zur römischen Macht entwickeln konnte. Dieses Zentrum der jüdischen Religion erneut entstehen zu lassen, konnte nicht in seinem Interesse sein. Vgl. allgemein dazu Schäfer 2003, $1 \mathrm{ff}$.

17 Ritti 2004, 297-340 = SEG 55, $1416=$ AE 2004, $1424=$ Ritti 2017, 386-388. Vgl. auch Jones 2018, 922.

$18 \mathrm{Zu}$ den Reisen Hadrians in dieser Zeit: Halfmann 1986, 192 f.; Halfmann 2019, 235-248.

19 Siehe Bronzemünzen der colonia Aelia Capitolina, die auf der Rückseite den Kaiser zeigt, der mit dem Ochsengespann die Grenze des Pomeriums pflügt: COL AEL KAPIT // COND (RPC III Nr. 3964). Auch auf Münzen von Caesarea, die unter Hadrian geprägt wurden, findet sich diese Szene (RPC III Nr. 3958). Dort fehlt allerdings das Wort COND; dieser Zusatz bei Aelia Capitolina lässt erkennen, dass das Bild nicht nur generell auf den Koloniestatus verweist wie bei Caesarea, sondern auf den konkreten Akt der Gründung, der betont werden soll.

20 Dieser wichtige Hinweis bei Birley 1997, 231-233.

$21 \quad$ P. Yadin $42=5 / 6$ Hev. 42. 
auf das Frühjahr 132 führt. ${ }^{22}$ Damit aber muss die Kolonie bereits vor dem Frühjahr 132, als der Aufstand ausbrach, existiert haben. Wie weit die ersten Schritte zur Einrichtung der neuen Stadt damals bereits getan waren, ist dabei irrelevant. Der Gründungsakt war für Bar Kochba und seine Anhänger entscheidend.

Wer gehörte zu den ersten Bürgern der neuen Kolonie? Wenn man davon ausgeht, dass sich in den 60 Jahren, seit die legio X Fretensis in Jerusalem lag, manche der Veteranen, die aus dieser Einheit ausschieden, dort niedergelassen und Familien gegründet hatten, dann sollten sie wohl unmittelbar in die colonia eingegliedert worden sein. Doch vor allem wird man wohl davon ausgehen können, dass Soldaten, die eben zum Zeitpunkt der Koloniegründung oder in den Jahren danach in den Veteranenstatus entlassen wurden, dort angesiedelt wurden. Denn wäre die Bevölkerung in den canabae genügend groß gewesen, dann hätte Hadrian dort auch ein muncipium einrichten können, wie er es an nicht wenigen anderen Orten durchgeführt hat. ${ }^{23}$ Einen Hinweis auf diese Ansiedlung von ausgedienten Militärs darf man wohl in dem Vexillum und dem Legionsadler sehen, die auf hadrianischen Münzen der colonia zu sehen sind; das vexillum erscheint dabei auf den Bronzen, die zeigen, wie der rituelle sulcus um die künftige Stadt gezogen wird. ${ }^{24}$ Beides gehört offensichtlich zusammen.

Konkret lassen sich allerdings Veteranen aus Legionen, aber ebenso aus den Auxilien der Provinz im Einzugsbereich der colonia kaum nachweisen. Soweit wir aus dem Bereich des heutigen Israel Inschriften von Veteranen von konkreten Plätzen kennen, liegen diese - vielleicht mit einer oder zwei Ausnahmen - so weitab von Jerusalem, dass diese mit der Neugründung nicht in Verbindung gebracht werden können. ${ }^{25}$ Lediglich bei einem Grab, das ein ehemaliger decurio einer Auxiliarkohorte bei Betholetepha-Beit Nattif, ca. $35 \mathrm{~km}$ südwestlich von Jerusalem, für sich und seine Frau errichtete, könnte man einen Zusammenhang mit der Kolonie vermuten. ${ }^{26}$ Doch muss offen bleiben, ob der Ort zur Zeit der Erbauung des Grabes noch auf dem Territorium der hadrianischen Neugründung lag. ${ }^{27}$ An einigen anderen Orten hat man lateinische, meist fragmentarische Inschriften gefunden, die aber nicht von Veteranen sprechen. Das ist etwa bei Gophna, mehr als $20 \mathrm{~km}$ nördlich von Jerusalem ${ }^{28}$ der Fall oder auch bei Beit Jimal, nicht weit von Betholetepha entfernt. ${ }^{29}$ Latein wurde - das lässt sich klar zeigen - in der Provinz fast ausschließlich von Personen verwendet, die eng mit Rom und seinen Herrschaftsinteressen verbunden waren; das aber waren meist Soldaten und vielleicht deren Angehörige. So könnte es durchaus sein, dass diese Reste lateinischer Inschriften auf ausgediente

22 So schon vor vielen Jahren Millar 1993, 546.

23 Siehe Zahrnt 1991, 463-486.

$24 R P C$ III Nr. 3964. Birley 1997, 231 f. verweist auf die Kinder, die neben der Personifikation der $I u$ daea auf den Adventusmünzen (BMC III 493 f.) für die Provinz erscheinen und vermutet, sie könnten auf die Gründung der Kolonie verweisen.

25 Siehe die verschiedenen Listen von Inschriften von aktiven Soldaten und Veteranen, die in lateinischer und griechischer Sprache abgefasst sind, bei Eck 2016, 127-140.

26 CIIP IV 1, 3297.

27 So nach Kloner - Klein - Zissu 2017, 131-141, hier 134. Dieser Artikel ist die vollständige Fassung dessen, was die Autoren schon in einem Kongressbericht vorgelegt hatten: Zissu - Klein - Kloner 2014, 219-223.

28 CIIP IV 1, 2685.

${ }^{29}$ CIIP IV 1, 3191. 
Militärs verweisen, die sich dort niedergelassen hatten und bestattet wurden, wie es vor allem bei dem Fragment aus Beit Jimal naheliegt. Träfe dies zu, dann könnte sich das Territorium von Jerusalem bis dorthin erstreckt haben. Doch bleiben solche Schlüsse notwendigerweise äußerst unsicher.

Lediglich eine einzige lateinische Grabinschrift darf man mit Sicherheit mit einer Ansiedlung von Soldaten in Verbindung bringen. Nach dem Text ist eine Valeria Sedata, die Tochter eines L. Valerius, im Alter von vier Jahren bei Moza, ca. $10 \mathrm{~km}$ westlich von Jerusalem bestattet worden. ${ }^{30}$ Dort aber waren bereits kurz nach der Zerstörung Jerusalems noch von Vespasian 800 Veteranen angesiedelt worden, ohne dass diese damals aber eine Gemeinde gebildet hätten. ${ }^{31}$ Wenn diese Ansiedlung die 60 Jahre bis zur Gründung der colonia überlebt hat, dann sind deren Bewohner, zumindest diejenigen, die römische Bürger waren, ebenso in die neu gegründete Stadt aufgenommen worden wie die Römer, die sich in den canabae beim Legionslager niedergelassen haben.

Bei der Gründung der Kolonie sollten jedenfalls Veteranen dort angesiedelt worden sein. Vorstellbar ist auch, dass nach dem Ende des Bar Kochba Krieges Soldaten der Legionenabteilungen, die für die Kämpfe eigens aus anderen Provinzen nach Iudaea abgeordnet worden waren, in die Kolonie aufgenommen wurden. Man kann annehmen, dass während der Kämpfe manche zwar ihre normale Dienstzeit erreicht hatten, aber dann noch für einige zusätzliche Jahre bei der kämpfenden Truppe zurückbehalten wurden, ${ }^{32}$ was in solchen Situationen verständlich war. Umso mehr Soldaten wurden nach dem Abschluss des Krieges entlassen; ihnen als Entlassungsprämie Land auf dem Territorium der neuen colonia Aelia Capitolina zuzuweisen, erscheint durchaus realistisch. Dass nach den massiven Verlusten in der jüdischen Bevölkerung in der Provinz viel herrenloses Land vorhanden war, das für Veteranen verwendet wurde, ist durch einen Papyrus aus Caesarea bekannt, der von einer Gerichtsverhandlung des Jahres 152 n.Chr. berichtet, in der es um eine solche Landanweisung ging. ${ }^{33}$ Das Verfahren betraf einen Veteranen, der in der Peraia Land erhalten hatte. Solches sollte auch in der colonia Aelia Capitolina geschehen sein, zumal gerade das Herzland des jüdischen Volkes im Zentrum der Kämpfe gestanden hatte, in dem schließlich nach 136 für lange Zeit keine jüdischen Siedlungen mehr existierten. Land stand also in großem Umfang zur Verfügung. Es wäre überraschend, wenn der römische Fiskus dieses nicht für die entlassenen Soldaten verwendet hätte; das sparte Entlassungsgelder. Dass die kaiserliche Administration nicht nur die Veteranen der beiden Legionen der Provinz, sondern auch Abteilungen zahlreicher anderer Legionen nach den langjährigen Kämpfen in die Bürgerschaft der colonia aufgenommen

30 CIIP I 2, 748; siehe Kloner - Klein - Zissu 2017, 137 mit einem irrigen Verständnis der Filiation. Dass man in einer Publikation des Jahres 2017 nicht die richtige Lesung in CIIP I 2, 748 (erschienen 2012) verwendet hat, ist etwas irritierend. Die Datierung ans Ende des 2., Anfang des 3. Jh. auf Grund der Buchstabenformen ist ohne jede Basis, da das Vergleichsmaterial dazu fehlt. Wenn dort auch CIIP I 2, 735, der Grabstein eines wohl thrakischen Auxiliarsoldaten, ebenfalls im Kontext der Bestimmung des Jerusalemer Territoriums angeführt wird, dann ist den Autoren entgangen, dass hier ein Soldat während des Dienstes gestorben ist. Damit kann dessen Grabstein in der Nähe von Abu Gosh mit der Frage nach dem Territorium der colonia Aelia Capitolina in keiner Weise etwas zu tun haben.

31 Iosephus, bell. Iud. 7, 217.

32 Siehe die Formel quinque et viginti pluribusve stipendis emeritis in den Diplomen der Auxiliartruppen.

33 Eck 1998, 249-255 = Eck 2014, 266-274; Eck 2000, 139-148 = Eck 2014, 275-283. 
hat, ist ein nicht ganz fern liegender Gedanke. In der konkreten Überlieferung hat dies aber - bisher - keinen Niederschlag gefunden.

Allerdings ist auch zu berücksichtigen, dass offensichtlich nicht alle Veteranen, die in der Provinz entlassen wurden, sich dort niederlassen wollten. Ein weiterer Papyrus aus Caesarea, diesmal aus dem Jahr 150, zeigt nämlich, dass Veteranen, die bereits in der Anfangszeit des Bar Kochbakrieges aus der Flotte von Misenum in die legio X Fretensis versetzt worden waren, nicht gewillt waren, in der Provinz zu bleiben. Sie waren in Ägypten für die Flotte von Misenum ausgehoben worden, wollten aber nun nach siebzehn Jahren Dienst in Judaea und dann Syria Palaestina wieder in ihre Heimat zurückkehren. ${ }^{34}$ Was das Motiv für diesen Wunsch war, steht nicht in dem Dokument; es können sehr verschiedene Gründe dabei ausschlaggebend gewesen sein. Doch ist ebenso vorstellbar, dass die Provinz nach den langjährigen, oft brutalen Kämpfen mit den fanatisierten Rebellen Bar Kochbas den Veteranen nicht als eine attraktive Heimat erschienen ist und sie es deshalb vorzogen, nach 25-jährigem Dienst in Flotte und Legion, beide Male außerhalb Ägyptens, dorthin zurückzukehren. Das lässt sich vielleicht durch eine Beobachtung bei den Auxiliartruppen von Syria Palaestina erhärten. Die Soldaten dieser Einheiten erhielten wie überall im Reich nach der ehrenvollen Entlassung das römische Bürgerrecht, das ihnen durch kaiserlichen Erlass geschenkt und durch individuelle diplomata militaria bestätigt wurde. In allen Provinzen wurde ein Teil der Diplome innerhalb der Provinz gefunden, in der deren Empfänger früher gedient hatten, während ein Teil der Diplome in anderen Provinzen entdeckt wurde. Für die Truppen aus Syria Palaestina kennen wir mindestens 13 Diplome, die nach dem Ende der Rebellion im Jahr 136 an einzelne Auxiliare ausgegeben wurden. Das Auffällige daran ist aber, dass nur zwei von diesen in der Provinz selbst erhalten blieben, ${ }^{35}$ alle anderen wurden in anderen Provinzen gefunden, meist wohl im Donauraum oder in Kleinasien. Dieses Verhältnis entspricht in keiner Weise dem, was wir sonst im Imperium kennen. Deshalb liegt der Gedanke nicht so ganz fern, dass die Mehrheit der Hilfstruppenveteranen sich bewusst nicht in dieser Provinz niederließ, sondern lieber in eine Heimat zurückkehrte, die den meisten von ihnen nach 25 oder mehr Dienstjahren vermutlich auch fremd geworden war. ${ }^{36}$ Dennoch: die Heimat mochte ihnen gastlicher erschienen sein als Syria Palaestina, mit dem man schwerste Kämpfe verband. Auch die colonia Aelia Capitolina könnte von einer solchen Sichtweise der Veteranen auf die Attraktivität eines Lebens in dieser Provinz betroffen worden sein.

Wie weit sich das Territorium der colonia erstreckte, lässt sich nicht näher erkennen, wie schon die vorausgegangenen Hinweise auf Veteranen des Heeres gezeigt haben. Auch weitere archäologische Hinweise können diese Erkenntnis nicht leisten, obwohl das versucht wurde. ${ }^{37}$ Erkennbar sind bauliche Strukturen, die römischen Vorbildern folgen; doch sind solche nicht notwendigerweise auf das Landgebiet beschränkt, das unter der Jurisdiktion der Koloniemagistrate stand. Das Gebiet, das die Autoren auf diese Weise als Kolonieterritorium ausweisen, dürfte durchaus dazu gehört haben, doch ist es relativ klein; denn fast alle konkreten Ausgrabungsbefunde liegen in einem Radius von

34 PSI 1026 = CIL XVI App. Nr. 13 = Corpus Papyrorum Latinorum, Nr. 117.

35 RMD I $69=$ CIIP IV 2, 3827; und CIL XVI 87.

36 Siehe Eck 2012, 249-265 = Eck 2014, 229-244, hier 243 f.

37 Siehe den Artikel von Kloner - Klein - Zissu 2017. 
kaum mehr als zehn Kilometern um das Koloniezentrum. ${ }^{38}$ Das aber wäre eine sehr beschränkte Ausstattung der colonia gewesen. So können, da es kein Ausschlusskriterium gibt, auch Befunde außerhalb dieses Umkreises Teil des Bodens der Kolonie gewesen sein. ${ }^{39}$ Auch der Verweis auf die Entfernungsangaben auf Meilensteinen sowie im Onomastikon Eusebs führt nicht weiter, wie längst deutlich gemacht wurde. ${ }^{40}$ Falls der Jurisdiktionsbereich des Bischofs von Jerusalem im 4./5. Jh. n.Chr. einen Hinweis darauf geben kann, dann sollte sich die Kolonie sehr viel weiter erstreckt haben, als es zuletzt von archäologischer Seite angenommen wurde. ${ }^{41}$ Doch bedürfte dies einer detaillierten Einzeluntersuchung, die hier nicht geleistet werden kann.

Von der inneren Struktur und der konkreten Organisation dieser neuen Gemeinde wissen wir insgesamt nur sehr wenig. Denn die Quellenlage ist im Vergleich zu der Zeit vor der ersten großen Revolte und in gewisser Weise auch für die Spätantike äußerst schlecht. Eine auswertbare literarische Überlieferung gibt es nicht. ${ }^{42}$ Alles, was wir erkennen können, beruht allein auf den Inschriften und in sehr beschränktem Umfang auf den Münzen. Die Zahl der Inschriften aber ist für die fast zwei Jahrhunderte bis Konstantin sehr gering, was nicht so sehr verwunderlich ist; denn die Christianisierung Jerusalems seit dem 4. Jh. hat zu einer sehr tiefgreifenden baulichen Veränderung der Stadt und zu einer Zerstörung oder Wiederverwendung früherer Inschriften geführt. So ist die geringe Zahl von Inschriften aus dem 2. und 3. Jh. nicht so überraschend: im CIIP I 2 sind es nur insgesamt 79, die meisten in lateinischer Sprache. ${ }^{43}$

Was man von der inneren Struktur erkennen kann, entspricht im Wesentlichen dem, was man bei einer colonia civium Romanorum erwarten kann.

Bezeugt ist der ordo decurionum, der Rat der Stadt, der schon auf einer Statuenbasis für Antoninus Pius steht, ${ }^{44}$ einer der frühesten Inschriften der neuen Kolonie. Wie hoch das persönliche Vermögen der Mitglieder des Rates sein musste, ist unbekannt, ebenso wie viele Dekurionen es überhaupt gab. Sie hatten die üblichen Magistrate der Kolonie zu stellen, die IIviri, aediles, quaestores und quinquennales. Kein einziger Amtsträger aber ist bisher in einem epigraphischen Text erwähnt, was den enormen Verlust an Inschriften deutlich macht. Denn Personen der Führungsschicht einer Stadt hatten stets

38 Siehe die Karte bei Kloner - Klein - Zissu 2017, 132.

39 Auch die schon vorrömische Einteilung in Toparchien, die in römischer Zeit mit Veränderungen weiterlief, könnte bei der Zuweisung des Kolonieterritoriums berücksichtigt worden sein. Siehe allgemein Cotton 1999, 75-91, bes. 81-89.

40 Siehe prägnant zur Frage, wieweit Angaben auf Meilensteinen etwas zu den Territorien von Gemeinden sagen oder besser nicht sagen können: Isaac 1998, 48-75, bes. 62-65. Siehe auch sein Urteil zur Auswertung des Onomastikon des Eusebius für das Territorium von Jerusalem am Beginn des 4. Jh. in seiner Besprechung von Freeman-Grenville - Chapman III - Taylor 2003 und Steven Notley - Safrai 2005 in Henoch 29, 2007, 167-172, wo er darauf hinweist, dass die Autoren ,reach ... conclusions that are full of internal inconsistencies and can only be described as fanciful.“

41 Siehe dazu die Hinweise von Christoph Birkner Die Bedeutung Jerusalems für das Mönchtum der Judäischen Wüste: Monastische Topographie im hagiographischen Corpus Kyrills von Skythopolis (in Druckvorbereitung - ich danke Herr Birkner, dass er mir den Text schon vor der Drucklegung zugänglich gemacht hat).

42 Außer über die Errichtung eines Tempels für die kapitolinische Trias; siehe dazu zuletzt Newman $2017,37 \mathrm{ff}$.

43 Siehe dazu auch Isaac 2017, 331-343, bes. $341 \mathrm{ff}$.

44 CIIP I 2, 718. 
mehr Chancen, in einem inschriftlichem Denkmal zu überleben. IIviri kann man daraus erschließen, dass von pecunia publica gesprochen wird, mit denen Ehrenstatuen und Ehrenbögen mit ihren Statuen errichtet wurden ${ }^{45}$ Öffentliche Mittel aber mussten vom Dekurionenrat genehmigt werden, damit die beiden höchsten Amtsträger sie verwenden konnten.

Der römische Charakter der Stadt aber tritt dennoch sehr deutlich in den Inschriften hervor, die dem 2. und 3. Jahrhundert zugewiesen werden. Denn für die überwiegende Mehrheit aller Texte, die in CIIP II 2 für diese Epoche verzeichnet sind, insgesamt 56 von 79, wird die lateinische Sprache verwendet, nur für 23 die griechische. Wenige Neufunde seit der Publikation von Band I 2 des CIIP haben daran nichts geändert. ${ }^{46}$ Doch nicht die Zahl allein ist entscheidend; bedeutsam ist vielmehr, dass in allen, im engeren Sinn öffentlichen Inschriften, sei es an Bauten oder in Texten unter Ehrenstatuen, nur Latein erscheint. Griechisch ist dagegen neben einigen religiösen Inschriften völlig auf den privaten Bereich beschränkt. ${ }^{47}$

Diese Sprache verschwindet jedoch wie beispielsweise auch in Caesarea Maritima völlig seit dem 4. Jh. Die innere Struktur der Stadt hat sich dadurch grundsätzlich nicht verändert, wozu es auch keinen Grund gab. Diese lebte weiter wie der Name Aelia, der auch in christlichen Texten weiterhin erscheint. Symptomatisch ist vielleicht, dass in Eusebs Onomastikon, in dem es um die Orte geht, die im Alten und Neuen Testament erscheinen, nur ein einziges Mal der Name Jerusalem erscheint, ${ }^{48}$ an allen anderen Stellen aber nur Aìía. Vielleicht hat dieser Name auch damals noch gelegentlich an den Gründer erinnert.

\section{LITERATURVERZEICHNIS}

Avner, R., Greenwald, R., Ecker, A., Cotton, H. M. (2014), A New-Old Monumental Inscription from Jerusalem Honoring Hadrian, New Studies in the Archaeology of Jerusalem and Its Region 8: 96-101. Benoit, P. (1957), Une reconnaissance de dette du $\mathrm{II}^{\mathrm{e}}$ siècle en Palestine, in: Studi in onore di Aristide Calderini e Roberto Paribeni, Bd. II, Mailand: 257-272.

Bernini, A. (2018), Un riconoscimento di debito redatto a Colonia Aelia Capitolina, Zeitschrift für Papyrologie und Epigraphik 206, 183-193.

Bernini, A. (2019), New Evidence for Colonia Aelia Capitolina (P. Mich. VII $445+$ inv. 3888c + inv. 3944k), in: Proceedings of the $28^{\text {th }}$ International Congress of Papyrology, Barcelona: 557-562.

Birley, A. R. (1997), Hadrian: The Restless Emperor, London.

45 CIIP I 2, 718. 719. 720.

46 Am wichtigsten ist eine Ergänzung zu CIIP I 2, 715: Avner et al. 2014, 96 ff., sowie ein Fragment mit den sehr großen Buchstaben [--]NO PRAE[--]: Gendelman - Chalaf 2016, 115-127 (Hebr.).

47 Dabei ist es bei manchen Texten nicht eindeutig zu bestimmen, ob alle wirklich in die Epoche bis zum Ende des 3. Jh. gehören oder nicht vielleicht doch später datiert werden können; gleiches gilt umgekehrt für einige griechische Text, die statt ins 4. vielleicht noch ins 3. Jh. gehören. Für die öffentliche Erscheinung der Stadt ist das jedoch unerheblich.

48 Siehe Timm 2005, 45, Zeile 1; für die Form Aỉ $\lambda$ ía siehe S. 119 des Index. 
Cotton, H. M. (1999), Some Aspects of the Roman Administration of Judaea/Syria Palaestina, in: W. Eck (hg.), Lokale Autonomie und römische Ordnungsmacht in den kaiserzeitlichen Provinzen, München: 75-91.

Cotton, H. M. (2007), The Administrative Background to the New Settlement Recently Discovered near Giv'at Shaul, Ramallah-Shu'afat Road, New Studies in the Archaeology of Jerusalem 1: 12*-18*.

Cotton, H., Eck, W. (2002), P. Murabba' at 114 und die Anwesenheit römischer Truppen in den Höhlen des Wadi Murabba'at nach dem Bar Kochba Aufstand, Zeitschrift für Papyrologie und Epigraphik 138: $173-183$.

Dąbrowa, E. (1993), Legio X Fretensis: A Prosopographical Study of Its Officers (I-III c. A.D.), Stuttgart.

Eck, W. (1998), Ein Prokuratorenpaar von Syria Palaestina in P. Berol. 21652, Zeitschrift für Papyrologie und Epigraphik 123, 249-255.

Eck, W. (2000), Der Bar Kochba Aufstand, der kaiserliche Fiscus und die Veteranenversorgung, Scripta Classica Israelica 19: 139-148.

Eck, W. (2012), Der Bar Kochba-Aufstand der Jahre 132-136 und seine Folgen für die Provinz Judaea/ Syria Palaestina, in: P. Urso (hg.), Iudaea socia - Iudaea capta, Pisa: 249-265.

Eck, W. (2014), Judäa - Syria Palästina. Die Auseinandersetzung einer Provinz mit römischer Politik und Kultur, Tübingen.

Eck, W. (2016), Soldaten und Veteranen des römischen Heeres in Iudaea/Syria Palaestina und ihre inschriftlichen Zeugnisse, in: C. Cordoni, G. Langer (hg.), "Let the Wise Listen and Add to Their Learning" (Prov 1:5). Festschrift for Günter Stemberger on the Occasion of His $75^{\text {th }}$ Birthday, Berlin: $127-140$.

Freeman-Grenville, G. S. P., Chapman III, R. L., Taylor, J. E. (2003), The Onomasticon by Eusebius of Caesarea, Jerusalem.

Gendelman, P., Chalaf, O. (2016), Excavations of the Strauss Complex in the Western Wall Plaza: A Preliminary Report on Building G, New Studies in the Archaeology of Jerusalem and Its Region 10: 115-127 (Hebr.).

Halfmann, H. (1986), Itinera principum. Geschichte und Typologie der Kaiserreisen im Römischen Reich, Stuttgart.

Halfmann, H. (2019), Hadrians Reisen: Alte und neue Erkenntnisse, in: K. Harter-Uibopuu (hg.), Epigraphische Notizen. Zur Erinnerung an Peter Herrmann, Stuttgart: 235-248.

Isaac, B. (1998), Milestones in Judaea: From Vespasian to Constantine, in: B. Isaac, The Near East under Roman Rule, Leiden: 48-75.

Isaac, B. (2017), Caesarea-on-the-Sea and Aelia Capitolina: Two Ambiguous Roman Colonies, in: C. Brélaz (hg.), L'héritage Grec des colonies Romaines d'Orient. Interactions culturelles dans les provinces hellénophones de l'empire romain, Paris: 331-343.

Jones, C. P. (2018), The Institutional History of Hierapolis, Journal of Roman Archaeology 31: 921 927.

Kloner, A., Klein, E., Zissu, B. (2017), The Rural Hinterland (territorium) of Aelia Capitolina, in: G. Avni, G. D. Stiebel (hg.), Roman Jerusalem: A New Old City, Portsmouth, RI: 131-141.

Millar, F. (1993), The Roman Near East, 31 BC-AD 337, Cambridge, MA-London.

Newman, H. I. (2017), The Temple Mount of Jerusalem and the Capitolium of Aelia Capitolina, in: G. C. Bottini, L. D. Chrupcała, J. Patrich (hg.), Knowledge and Wisdom: Archaeological and Historical Essays in Honour of Leah Di Segni, Jerusalem: 35-42.

Ritti, T. (2004), Documenti adrianei da Hierapolis di Frigia: le epistole di Adriano alla città, in: S. Follet (hg.), L'Hellénisme d'époque romaine. Nouveaux documents, nouvelles approches (1 ${ }^{e r}$ s. a.C.-III s. p.C.), Paris: 297-340.

Ritti, T. (2017), Hierapolis di Frigia IX: Storia e istituzioni di Hierapolis, Hierapolis-Istanbul.

Schäfer, P. (2003), Bar Kokhba and the Rabbis, in: P. Schäfer (hg.), The Bar Kokhba War Reconsidered: New Perspectives on the Second Jewish Revolt against Rome, Tübingen: 1-22. 
Steven Notley, R., Safrai, Z. (2005), Eusebius, Onomasticon: A Triglott Edition with Notes and Commentary, Leiden.

Timm, S. (2005), Das Onomastikon der biblischen Ortsnamen, Edition der syrischen Fassung mit griechischem Text, englischer und deutscher Übersetzung, Berlin.

Weksler-Bdolah, S. (2014), The Foundation of Aelia Capitolina in Light of New Excavations along the Eastern Cardo, IEJ 64: 38-62.

Zahrnt, M. (1991), Zahl, Verteilung und Charakter der hadrianischen Kolonien (unter besonderer Berücksichtigung von Aelia Capitolina), Stuttgarter Kolloquium zur historischen Geographie des Altertums 2, 1984 und 3, 1987, Bonn: 463-486.

Zissu, B., Klein, E., Kloner, A. (2014), Settlement Processes in the territorium of Roman Jerusalem (Aelia Capitolina), in: J. M. Alvarez, T. Nogales, I. Roda (hg.), XVIII CIAC: Centre and Periphery in the Ancient World, Merida: 219-223. 